\title{
Quantum dots in magnetic fields: thermal response of broken symmetry phases
}

\author{
D.J. Dean ${ }^{1,2}$, M.R. Strayer ${ }^{1,2}$, and J.C. Wells ${ }^{1,3}$ \\ ${ }^{1}$ Center for Engineering Science Advanced Research \\ Oak Ridge National Laboratory, Oak Ridge, Tennessee 37831 USA \\ ${ }^{2}$ Physics Division, Oak Ridge National Laboratory, Oak Ridge, Tennessee 37831 USA \\ ${ }^{3}$ Computer Science and Mathematics Division, Oak Ridge National Laboratory, Oak Ridge Tennessee 37831 USA
}

\begin{abstract}
We investigate the thermal properties of circular semiconductor quantum dots in high magnetic fields using finite temperature Hartree-Fock techniques. We demonstrate that for a given magnetic field strength quantum dots undergo various shape phase transitions as a function of temperature, and we outline possible observable consequences.
\end{abstract}

The quantum mechanical characteristics (i.e. ground- and excited-state energy levels) of semiconductor quantum dots were recently investigated as a function of increasing magnetic field and electron number [1.22. Unique structures that break rotational symmetry within a quantum dot and develop as a function of increasing magnetic field were also reported in theoretical investigations [3] and associated with experimental observations of charge-density redistribution 3.4. In this paper, we explore the thermal characteristics of the broken-symmetry phase of the charge-density distribution, showing that temperature can be used to induce transitions between phases of differing spatial symmetry.

Thermal properties of, and transitions in, strongly correlated quantum mechanical systems have been studied in various contexts. Perhaps the most obvious are the normal-conducting to super-conducting transitions in condensed matter systems. Many nuclear systems exhibit an intrinsic deformation in their ground states, and deformed-tospherical transitions have been studied in many models (see for example Refs. [5]). As a result, observable consequences, such as enhanced moments of inertia, decreasing quadrupole transition moments, and decreasing correlated pair-transfer amplitudes may be investigated.

In studies of many-body phenomena in quantum dots, experimental efforts have focused on mapping the magnetic field dependence of their ground-state structure by measuring the chemical potential via capacitance spectroscopy [8]. Cusps and steps in the chemical potential were found to clearly separate different ranges of magnetic fields [8, 1 ]. These features were identified with phase transitions in the charge density of the quantum dot. At magnetic field strengths on the order of a few tesla, all electrons become spin-polarized initiating the maximum density droplet (MDD) phase [8], in which the density is constant and homogeneous at the maximum value that can be reached in the lowest Landau level. The stability of the MDD is determined by a competition among the kinetic energy, external confinement, the Coulomb repulsion between electrons, and the attraction created by the Coulomb exchange term. For increasing magnetic field, the charge-density distribution of the droplet reconstructs $[9]$ with a ring of electrons breaking off from the MDD phase. This edge reconstruction has been shown via mean-field theory [3] calculations to result from a rotational symmetry-breaking phase transition from the MDD to a Wigner molecule or Wigner crystal phase. These calculations are in good qualitative agreement with recent experimental results where instabilities of the MDD state and other transitions in the high magnetic field region were accompanied by a redistribution of the charge density $[$.

Most of the experimental investigation of many-body phenomena in quantum dots was performed at very low temperatures on the order of $100 \mathrm{mK}$ and focused on the evolution of the ground state of the quantum dot as a function of increasing magnetic field and electron number [11, 12]. The thermal characteristics of these systems are largely unexplored, especially for large values of the magnetic field. We explore the thermal response of the brokensymmetry phase at constant magnetic field and electron number and demonstrate that transitions among a number of distinct, broken-symmetry phases occur as the temperature is increased.

In describing the ground-state and low-lying (intra-band) excitations of the $N$-electron semiconductor nanostructures, it is often sufficient to restrict consideration to the conduction band using the effective-mass approximation [13]. We consider the problem of $\mathrm{N}$ electrons of effective mass $m^{*}$ in a plane, $(x, y)$, confined by an external parabolic potential, $V(r)=\frac{1}{2} m^{*} \omega_{0}^{2} r^{2}$, and subject to a strong magnetic field $\vec{B}=B_{0} \vec{e}_{z}$. We consider the Zeeman splitting but neglect the spin-orbit interaction. The Hamiltonian for such a system is

$$
\hat{H}=\sum_{i}\left[\frac{\left(\vec{p}-\frac{e}{c} \vec{A}\right)^{2}}{2 m^{*}}+V\left(r_{i}\right)+\frac{g^{*} \mu_{B}}{\hbar} \vec{B} \cdot \vec{S}_{i}\right]
$$




$$
+\sum_{i<j} \frac{e^{2}}{\varepsilon\left|\vec{r}_{i}-\vec{r}_{j}\right|}
$$

where the vector potential is $\vec{A}\left(\vec{r}_{i}\right)=\left(B_{0} / 2\right)\left(-y_{i}, x_{i}, 0\right), g^{*}=0.54, \varepsilon=12.9, m^{*}=0.067 m_{e}$, and $\hbar \omega_{0}=3 \mathrm{meV}$.

We solve this equation at the finite-temperature Hartree-Fock level. This is the initial step to account for electronelectron correlations within the quantum dot and suffices for a qualitative discussion of phase transitions and thermal behavior. Hartree-Fock is implemented across nuclear and atomic physics as a first step towards the solution of the quantum many-body problem [14]. Since the Hartree-Fock equations are nonlinear, the self-consistent potential obtained from their solution will not necessarily show the same symmetries as the original Hamiltonian. Of course, the exact wave will maintain the symmetries of the original Hamiltonian. As a point of reference, an estimate of the relative uncertainty in total angular momentum at high magnetic field strengths was shown to be roughly $10 \%$ by the authors of Ref. 10].

The equations describing the static mean field (i.e., Hartree-Fock) at finite temperature for an $N$-electron system are

$$
\left[\hat{K}+\hat{W}(\beta)-E_{\alpha}\right] \mid \phi_{\alpha}(\beta)=0
$$

where $E_{\alpha}$ are the single particle energies associated with the single-particle wave functions $\phi_{\alpha}$, and $\hat{K}$ is the one-body kinetic-energy operator. The one-body Hartree-Fock field, $\hat{W}(\beta)$, is obtained in terms of the Hamiltonian (1) and the one-body density matrix, $\hat{\rho}(\beta)$ as,

$$
\hat{W}(\beta)=\operatorname{Tr}[\hat{\rho}(\beta) \hat{H}] .
$$

The single-particle wave functions are denoted as $\left|\phi_{\alpha}(\beta)\right\rangle$, and $\beta=1 / k T$ is the inverse temperature. The representation of the one-body density is given in terms of the occupation numbers $n_{\alpha}(\beta), \rho(\beta)=\sum_{\alpha} n_{\alpha}(\beta)\left|\phi_{\alpha}(\beta)\right\rangle\left\langle\phi_{\alpha}(\beta)\right|$, and the Fermi-Dirac occupation numbers at chemical potential $\mu$ are $n_{\alpha}(\beta)=1 /\left[e^{\beta\left(E_{\alpha}-\mu\right)}+1\right]$. Note that Tamura and Ueda 15] studied the number fluctuations $\left\langle(\delta N)^{2}\right\rangle$ of a quantum dot as a function of $N$ for different field strengths and found that the fluctuations were rather small (on the order of 0.1) in finite-temperature Hartree-Fock applications. We expect qualitative agreement with experiment as Hartree-Fock is known to overestimate the magnetic field strength at which transitions occur 16, 12,3]. However, for a fixed field strength, experimental evidence of the thermal transitions should still be qualitatively visible.

We use a Fock-Darwin basis expansion to solve the finite-temperature Hartree-Fock equations. Since we use a high $(\approx 12 \mathrm{~T})$ magnetic field, we consider only angular momentum states with the $n=0$ principal quantum number. The electrons carry spin, and so our states are labeled by $k=\left\{l_{k}, s_{k}\right\}$, where $l_{k}$ is the angular momentum projection of the $k$-th state and $s_{k}$ is the spin of that state. We found convergence using fifty states for the $N \leq 8$ systems. We also checked our zero-temperature results with other publications [10] for various numbers of electrons in the dot and found satisfactory agreement.

We begin the discussion of our results by investigating the electron charge, angular momentum, and spin densities as a function of increasing temperature for the $N=6$ system at $B_{0}=12.15 \mathrm{~T}$. We show densities at representative temperatures of $3.87 \mathrm{~K}$ (the low temperature limit, $\nu=6$ ), $11.97 \mathrm{~K}$ (before the first phase transition, $\nu=6$ ), $13.65 \mathrm{~K}$ (in the second phase, $\nu=5$ ), and $14.32 \mathrm{~K}$ (in the third phase, $\nu=4$ ), where $\nu$ is the number of definable high-density regions (or vortices) in the charge density plots. The density, shown in Fig. 17a-d, begins as a fairly well-defined Wigner crystal at $3.87 \mathrm{~K}$, which exhibits some degree of thermal broadening at $11.97 \mathrm{~K}$. The $\nu=5$ and $\nu=4$ phases continue to show a similar amount of density in the remaining vortices, while the density of the thermally dissipated vortices have effectively spread through the entire dot. The angular momentum along the $B$-field direction, shown in Fig. 国-h, exhibits well-defined structures at low temperatures which tend to decrease rapidly as one moves through the various phases. Although the high charge density regions in the $\nu=4$ phase are still well defined, the angular momentum in this high-temperature phase has nearly washed out. Finally, we show in Fig. 11i-1 the spin density defined as $\rho_{s}(x, y)=\left[\rho_{\uparrow}(x, y)-\rho_{\downarrow}(x, y)\right] /\left[\rho_{\uparrow}(x, y)+\rho_{\downarrow}(x, y)\right]$, where $\rho_{\uparrow}\left(\rho_{\downarrow}\right)$ refer to the spin up (down) density. At these temperatures, little appreciable spin depolarization occurs and the spin density remains above 0.8 for the entire region where there is appreciable charge density.

The suddenness of the phase transitions seen in Fig. 1 become quite evident when the internal energy of the quantum dot is plotted as a function of the temperature. We show the three phases of the dot in Fig. 2a. Note that the $\nu=6$ phase exists as an excited configuration when the most probable Hartree-Fock solution is the $\nu=5$ phase. Similarly, the $\nu=4$ configuration exists as a possible excited configuration of the system even at fairly low temperatures. The specific heat, $C_{v}=d\langle H\rangle / d T$ (with $\mathrm{T}$ in units of $\mathrm{eV}$ ), is shown in Fig. 2 $\mathrm{b}$. Clearly, when the energy undergoes a phase 
transition, the specific heat shows a sharp structure. This occurs since the energy is piece-wise continuous along the three phases.

These calculations suggest that the quantum dot exhibits a band structure of many-body levels. The lowtemperature states all have the same intrinsic shape characteristic (the same vortex structure). As we increase the temperature, other $\nu$ phases become accessible. At the point when two bands of different intrinsic character cross in energy, we find a phase transition. Similar phenomena are found in nuclear physics, where at higher nuclear excitation energies the eigenstates of the system may be of a different intrinsic deformation when compared to states of the ground-state band [17].

We also find generally that the exchange energy decreases as a function of increasing temperature but with differing slopes in the different phases. In the region of phase transitions, the ratio of the exchange energy to the direct energy decreases from 0.38 (at $11.5 \mathrm{~K}$ ) to 0.32 (at $13 \mathrm{~K}$ ) in the $\nu=6$ phase. In the $\nu=5$ phase, this ratio decreases from 0.32 at $T=13 \mathrm{~K}$ to 0.28 at $T=13.8 \mathrm{~K}$, and from 0.28 at $T=13.8 \mathrm{~K}$ to 0.26 at $T=14.5 \mathrm{~K}$ in the $\nu=4$ phase. The slopes of the decreasing ratio are different across the three phases, with $\nu=6,5$ being the largest and $\nu=4$ more gradual.

The occupation probabilities of the Fock-Darwin states, $n_{F D}$, for the temperature conditions of Fig. 1 are shown in Fig. 2 $\mathrm{c}$. In the low-temperature phase $(3.9 \mathrm{~K}$, solid line), the $l=0$ state is occupied, while an edge reconstruction has occurred for the remaining five electrons. As we increase the temperature to $12 \mathrm{~K}$ (dotted line), we see a spreading of the occupations in both the low and high angular momentum channels with about 0.8 particles in the $l=0$ state. The $\nu=5$ phase brings a dramatic decrease of occupation in the $l=0$ state and a shift to lower angular momentum for the reconstructed edge. This trend continues after the $\nu=4$ transition.

Signatures of the density transitions that we have seen also appear in the Hartree-Fock occupations $n_{H F}$ as shown in Fig. 2 $\mathrm{d}$. At low temperatures $(3.9 \mathrm{~K})$ the familiar step-function behavior is evident from the figure. At $12 \mathrm{~K}$ in the $\nu=6$ phase, we see a decrease of occupation to roughly 0.8 in the lowest six Hartree-Fock levels and a spreading to higher energy states. As the system undergoes the transition to $\nu=5$, we see only five Hartree-Fock levels significantly filled (with $n_{H F}>0.7$ ), and finally in the $\nu=4$ phase, only four Hartree-Fock levels are significantly filled. The occupation number-spreading, which is due to thermal excitation of the system, is enhanced significantly when the system undergoes a phase transition.

The phase transitions that we have shown in the preceding discussion have definite observable consequences. In Fig. 3 a we plot the chemical potential, that is, the separation energy $\Delta(N, T)=E_{N}(T)-E_{N-1}(T)$ to remove a particle from the quantum dot at a given temperature. Since this is an energy difference, $\Delta(N, T)$ will be influenced by transitions within both the $N=6$ and $N=5$ systems, and we expect changes in slope at the transition points. The $\nu=5$ to $\nu=4$ transition in the $N=5$ dot occurs at roughly $12 \mathrm{~K}$, causing a sharp rise in $\Delta(N=6)$. A slope change in $\Delta(N=6)$ is seen at $\approx 13 \mathrm{~K}$, where the $\nu=6$ to $\nu=5$ transition occurs in the $N=6$ system. The decrease from $14-14.3 \mathrm{~K}$ occurs when the $N=6$ system makes the transition from $\nu=5$ to $\nu=4$. A final change in slope occurs when the $N=5$ dot makes the transition from $\nu=4$ to $\nu=3$. For the brief temperature interval when the $N=6$ and $N=5$ dots are in the same $\nu$ phase, we see a decrease in the chemical potential.

We observe similar changes in the inverse compressibility, $\Delta_{2}(N, T)=E_{N+1}(T)-2 E_{N}(T)+E_{N-1}(T)$. This quantity has been measured for quantum dots in low magnetic fields [18] and studied in Hartree-Fock theory for ground-state properties [19]. In our case, the $N=7,6$, and 5 dots participate in the observable. We again notice strong effects as one passes through transition points in either of the three systems contributing to the observable. Figure $3 \mathrm{~b}$ shows $\Delta_{2}(N=6)$ as a function of temperature. Before transitions occur, $\Delta_{2}$ remains fairly constant. A large decrease begins at $12 \mathrm{~K}$, where the $N=5$ system undergoes its first transition. Interestingly, $\Delta_{2}$ increases significantly when the $N=6$ and $N=5$ dots are in the $\nu=4$ phase.

In order to investigate the sensitivity of our results to the approximations made (namely the Hartree-Fock approximation) we extended our studies to include the second-order perturbative correction to the Hartree-Fock energy. This correction to the energy is given by

$$
\Delta E_{2}=\frac{1}{4} \sum_{a b \leq \epsilon_{F}} \sum_{r s>\epsilon_{F}} \frac{|\langle a b|\bar{v}| r s\rangle|^{2}}{\varepsilon_{a}+\varepsilon_{b}-\varepsilon_{r}-\varepsilon_{s}}
$$

where we restrict the sums to be below and above the Fermi energy surface, $\epsilon_{F}$. In this expression, the HartreeFock states are given by $a, b, r, s$, with associated single-particle energies $\varepsilon_{a}, \varepsilon_{b}, \varepsilon_{r}, \varepsilon_{s}$, and $\langle a b|\bar{v}| r s\rangle$ are the antisymmetrized two-body Hartree-Fock matrix elements of the original two-body interaction of the Hamiltonian. We note that the absolute value of $\Delta E_{2}$ in our calculations is always less than $1 \%$ of the total energy, and in fact only slightly changes the observable quantities, as is evident from Fig. 3, where the second-order perturbative results are also shown for $\Delta$ and $\Delta_{2}$. 
In addition to measurements of energy differences, one should be able to experimentally probe the thermal phase transitions using far-infrared spectroscopy and X-ray scattering. Far-infrared spectroscopy was used to investigate the excitations of InAs quantum dots as a function of the electron number per dot 20]. Grazing incidence X-ray scattering was recently used to generate a full structural characterization of quantum dots [21], including information on the elastic form factor. While these experiments were carried out at very low temperatures, it is conceivable that one could study the thermal response of quantum dots using X-ray or far-infrared scattering. A Fourier transform of the charge density produced in our calculations gives the elastic form factor that can be used to characterize the dot. We show in Fig. 1 the form factor, $|\rho(q)|$, as a function of the momentum transfer vector $|q|=\sqrt{q_{x}^{2}+q_{y}^{2}}$. A well-defined minimum is apparent at approximately $|q|=0.7 \mathrm{ev}^{-1}$ in all three phases. This first minimum is related to the size of the dot and clearly does not change in the three phases. This is apparent also from a close inspection

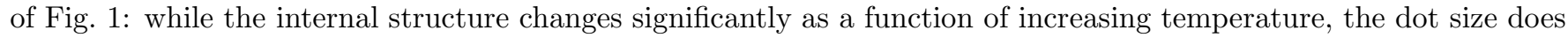
not change. The height of the second maximum slightly increases as in phase $\nu=5$ before decreasing in the $\nu=4$ phase. The position of the second minimum increases as a function of $q$ significantly and would be a distinguishing feature in X-ray or far-infrared scattering experiments used to probe the thermal phases of a quantum dot.

We see in the above calculations that quantum dots exposed to high magnetic fields undergo various phase transitions as the temperature increases. Indeed, we have followed the $N=6$ system through the $\nu=3$ and 2 phases as well. We have also studied these transitions at various magnetic-field strengths, and with differing numbers of electrons in the dot up to $N=20$, with the results presented here being characteristic of the general behavior. Our purpose in this paper has been to demonstrate that thermal excitations of a quantum dot induce phase transitions within the dot that should have observable consequences. Thus, we have shown that temperature may also be used as a control to tune the characteristics of quantum dots. Inclusion of the full many-body correlations may slightly dampen some of these effects [22], but signatures of the phase transitions should still appear in scattering experiments and measurements of the inverse compressibility and chemical potential of the quantum dot.

This research was sponsored by the Laboratory Directed Research and Development Program of Oak Ridge National Laboratory, under Contract No. DE-AC05-00OR22725 managed by UT-Battelle, LLC. by the U.S. Department of Energy.

[1] D.R. Stewart, D. Sprinzak, C.M. Marcus, C.I. Duruoz, and J.S. Harris, Science 278, 1784 (1997).

[2] L.P. Kouwenhoven, T.H. Oosterkamp, M.W.S. Danoesatro, M. Eto, D.G. Austing, T. Honda, and S. Tarucha, Science 278, 1788 (1997).

[3] S.M. Reimann, M. Koskinen, M. Manninen, and B.R. Mottelson, Phys. Rev. Lett. 83, 3270 (1999).

[4] T.H. Oosterkamp, J.W. Janssen, L.P. Kouwenhoven, D.G. Austing, T. Honda, and S. Tarucha, Phys. Rev. Lett. 82, 2931 (1999).

[5] F. Iachello, N.V. Zamfir, and R.F. Casten, Phys. Rev. Lett. 81, 1191 (1998).

[6] D.J. Dean, S.E. Koonin, K. Lanbanke, P.B. Radha, and Y. Alhassid, Phys. Rev. Lett. 74, 2909 (1995).

[7] J.A. White, S.E. Koonin, and D.J. Dean, Phys. Rev. C61, 34303 (2000).

[8] R. C. Ashoori, Nature 379, 413 (1996).

[9] C. de C. Chamon and X.G. Wen, Phys. Rev. B 49, 8227 (1994).

[10] H.-M. Müller and S.E. Koonin, Phys. Rev. B 54, 14532 (1996).

[11] E.B. Foxman, U. Meirav, P.L. McEuen, M.A. Kastner, O. Klein, P.A. Belk, D.M. Abusch, and S.J. Wind, Phys. Rev. B50, 14193 (1994).

[12] O. Klein, D. Goldhaber-Gordon, C. de C. Chamon, and M.A Kastner, Phys. Rev. B53, R4221 (1996).

[13] L. Jacak, P. Hawrylak, and A. Wójs, Quantum Dots, (Springer, Berlin, 1997).

[14] W. Satula, J. Dobaczewski, W. Nazarewicz, Phys. Rev. Lett. 81, 3599 (1998)

[15] H. Tamura and M. Ueda, Phys. Rev. Lett. 79, 1345 (1997).

[16] O. Klein, C.D. Chamon, D. Tang, M. Abusch, D.M. Magder, U. Meirav, X.G. Wen, M.A. Kastner, and S.J. Wind, Phys. Rev. Lett. 74, 785 (1995).

[17] D.G. Sarantites, D.R. LaFosse, M. Delvin, F. Lerma, V.Q. Wood, J.X. Saladin, D.F. Winchell, C. Baktash, C.H. Yu, P. Fallon, I.Y. Lee, A.O. Macchiavelli, R.W. MacLeod, A.V. Afanasjev, and I. Ragnarsson, Phys. Rev. C57, R1 (1998).

[18] U. Sivan, R. Berkovits, Y. Aloni, O. Prus, A. Auerbach, and G. BenYoseph, Phys. Rev. Lett. 77, 1123 (1996); S.R. Patel, S.M. Cronenwett, D.R. Stewart, A.G. Huibers, C.M. Marcus, , C.I. Duruoz, J.S. Harris, K. Campman, and A.C. Gossard, Phys. Rev. Lett. 80, 4522 (1998). 
[19] S. Levit, D. Orgad, Phys. Rev. B60, 5549 (1999).

[20] M. Fricke, A. Lorke, J.P. Kotthaus, G. Medeiros-Ribeiro, and P.M. Petroff, Europhys. Lett. 36, 197 (1996).

[21] T.H. Metzger, I. Kegel, R. Paniago, A. Lorke, J. Peisl, J. Schulze, I. Eisele, P. Schittenhelm, and G. Abstreiter, Thin Solid Films 336, 1 (1998).

[22] A.V. Filinov, M. Bonitz, and Yu. E. Lozovik, Phys. Rev. Lett. 86, 3851 (2001).

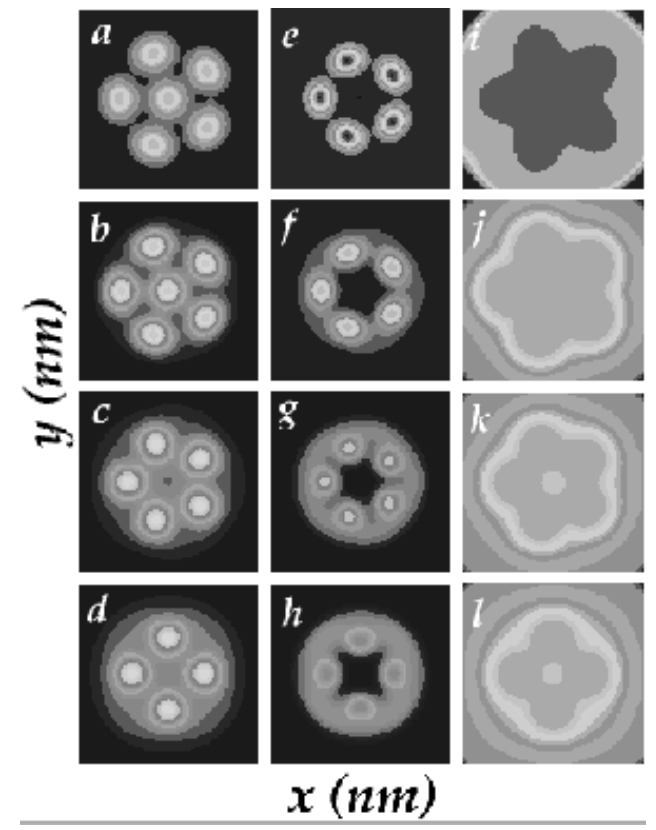

FIG. 1. Panels a-d, the charge density; panels e-h, the angular momentum density (in the $z$-direction); panels i-l, the spin density. In each panel, $-8 \mathrm{~nm} \leq x, y \leq 8 \mathrm{~nm}$.
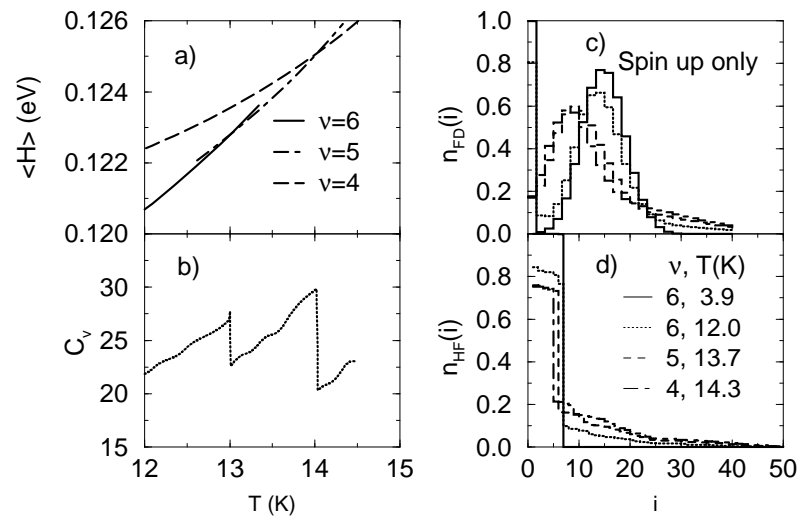

FIG. 2. a) Expectation value of the energy as a function of temperature showing the three phases as discussed in the text. b) The specific heat for the lowest-energy configuration of the quantum dot as a function of temperature. c) Occupation of the Fock-Darwin states. d) Occupation of the Hartree-Fock states. 


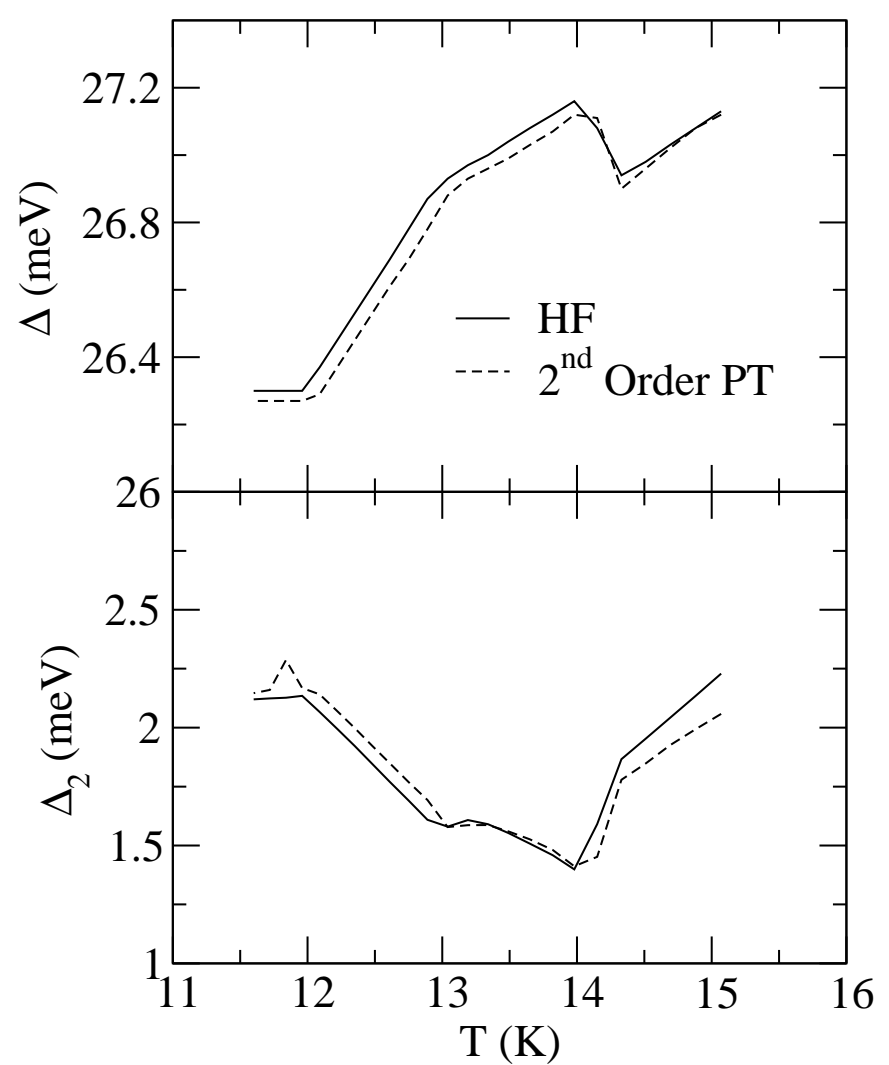

FIG. 3. a) Chemical potential, $\Delta$ and b) inverse compressibility, $\Delta_{2}$ for the $N=6$ system as a function of the temperature. The Hartree-Fock results are shown as a solid line, and the second-order perturbation theory results are given by the dashed line.

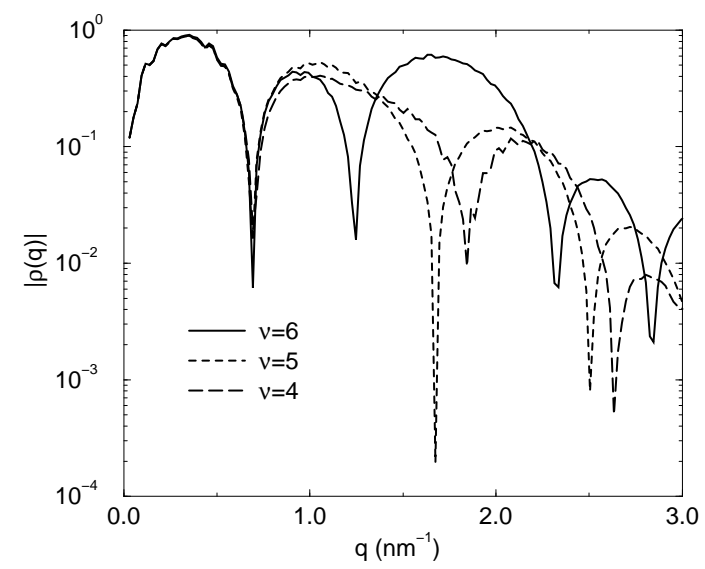

FIG. 4. The form factor in the three phases, $\nu=6(11.9 \mathrm{~K}), \nu=5(13.6 \mathrm{~K})$, and $\nu=4(14.3 \mathrm{~K})$. 